\title{
La « marmitte renversée » : construction discursive et fonctionnement argumentatif d'une insulte dans les polémiques des guerres de religion (1560-1600)
}

The "Overturned Pot": Discursive Construction and Polemic Use of an Insult during the French Religious Wars (1560-1600)

\section{Caroline Mellet et Paul-Alexis Mellet}

\section{(2) OpenEdition Journals}

\section{Édition électronique}

URL : http://journals.openedition.org/aad/1273

DOI : $10.4000 /$ aad. 1273

ISSN : 1565-8961

\section{Éditeur}

Université de Tel-Aviv

\section{Référence électronique}

Caroline Mellet et Paul-Alexis Mellet, « La « marmitte renversée » : construction discursive et

fonctionnement argumentatif d'une insulte dans les polémiques des guerres de religion (1560-1600) », Argumentation et Analyse du Discours [En ligne], 8 | 2012, mis en ligne le 15 avril 2012, consulté le 23 septembre 2019. URL : http://journals.openedition.org/aad/1273 ; DOI : 10.4000/aad.1273

Ce document a été généré automatiquement le 23 septembre 2019.

\section{cc) (†)}

Argumentation \& analyse du discours est mis à disposition selon les termes de la licence Creative Commons Attribution - Pas d'Utilisation Commerciale - Pas de Modification 4.0 International. 


\title{
La « marmitte renversée » : construction discursive et
} fonctionnement argumentatif d'une insulte dans les polémiques des guerres de religion (1560-1600)

\author{
The "Overturned Pot": Discursive Construction and Polemic Use of an Insult
} during the French Religious Wars (1560-1600)

Caroline Mellet et Paul-Alexis Mellet

\section{Introduction}

1 L'article présenté ici propose d'étudier une insulte au sein de la sphère religieuse et politique pendant les guerres de religion en France entre 1562 et 1598. Il s'agit de mettre en évidence les relations étroites entre les conditions historiques et discursives précises et la construction d'une insulte afin d'en cerner l'efficacité argumentative spécifique. Nous précisons néanmoins que nous laissons de côté ici l'étude, encore à faire, de la relation entre insulte et passage à l'acte. Nous étudions davantage un énoncé insultant en tant que «formule » selon la définition qu'en donne Alice KriegPlanque $^{1}$, fonctionnant comme un référent social discuté et favorisant les violences religieuses. Le contexte des guerres de religion est en effet très riche pour qui étudie les insultes. De fait, les tensions confessionnelles, particulièrement fortes dans l'Europe du 16e siècle, au moment même où se développe l'imprimerie, révèlent différents usages polémiques plus ou moins complexes. Il suffit de voir avec quelle insistance les édits de pacification interdisent l'usage des injures pour comprendre l'importance de leur diffusion ${ }^{2}$. 
2 Nous proposons ainsi de rendre compte rapidement du contexte politique et religieux des années 1560 au sein duquel va se développer le motif de la marmite. Cet aperçu justifiera la constitution du corpus. L'étude portera alors plus précisément sur la construction discursive du terme "marmite» comme insulte, ce qui permettra de rendre compte de certains aspects de son fonctionnement argumentatif.

\section{Le contexte historique de la polémique}

\subsection{Le contexte religieux des années 1560}

3 A partir de la réforme de Luther (vers 1520), suivie par celles de Zwingli, Bullinger et Calvin (vers 1530), les critiques à l'encontre du clergé séculier (prêtres, évêques) et du clergé régulier (ordres monastiques) se multiplient. On choisira ici d'exclure les polémiques vis-à-vis des juifs, des musulmans, des luthériens ainsi que des différents groupes minoritaires (antitrinitariens, anabaptistes, etc.), pour se concentrer sur celles opposant les catholiques aux calvinistes en France et dans certaines terres du SaintEmpire (Saxe, Palatinat, cantons suisses). On voit alors apparaître les années 1559-1562 comme cruciales. Comment expliquer ce phénomène?

Deux partis opposés s'organisent dans l'entourage du roi et cherchent à peser sur les décisions des fils d'Henri II, d'abord François II (qui hérite de la couronne en 1559 à 15 ans) puis Charles IX (qui lui succède en 1560 à dix ans). Les conflits sont vifs avec ceux des catholiques qui refusent la politique de concorde prônée par Catherine de Médicis. Mais c'est la question de la messe qui va progressivement cristalliser toutes les tensions. L'eucharistie devient le cœur de la différence théologique entre les confessions en présence : contre la doctrine catholique de la Cène, Calvin rejette la transsubstantiation (la présence réelle du corps et du sang du Christ), qui n'est à ses yeux qu'une invention de la papauté ${ }^{3}$ (Calvin 1536). Le différend est à son comble quand Théodore de Bèze, futur successeur de Calvin à Genève, lors d'un colloque organisé à Poissy (septembre 1561) pour tenter de trouver une solution à la crise, déclare dès l'ouverture que le corps et le sang du Christ sont totalement étrangers au pain et au vin. C'est la fin de l'espoir de conciliation entretenu par Catherine de Médicis et le chancelier Michel de L'Hospital ${ }^{4}$.

\subsection{Le motif de la marmite dans les écrits polémiques entre catholiques et réformés}

5 Se développe alors une culture complexe de l'imprimé, comprenant des genres aussi variés que des catalogues de martyrs (Histoire des martyrs de Jean Crespin, 1564), des recueils de prières mais aussi des satires croisées. Certains pamphlétaires catholiques comme Artus Désiré sont devenus célèbres en moquant la « singerie huguenotique » de «Monsieur de Baise » - la gueunon de Bèze - (Le Contrepoison, 1560). Mais le motif qui retient ici notre attention est celui de la marmite, qui est fondamental dans le cadre de la polémique liée à l'eucharistie. Il traduit en effet pour les protestants la gloutonnerie de ces moines cannibales, qui osent affirmer que la chair et le sang du Christ sont distribués rituellement et consommés collectivement tous les dimanches.

6 Si ce motif de la marmite a connu une si grande diffusion dans les années d'affirmation de la Réforme protestante, c'est aussi parce qu'il est l'héritier d'une longue tradition 
sacrée et profane. Il apparait à trois reprises dans les sources vétérotestamentaires, quand Jérémie a la vision d'un chaudron (Jérémie 1, 13-16), quand une marmite cuit la viande du peuple (Ezechiel 11, 1-12) et quand Jérusalem est comparée à une marmite rouillée (Ezechiel 24, 1-14). Mais, comme nous le verrons, seul le texte de Thomas BeauxAmis s'appuie largement sur ces sources. D'une façon plus générale, le chaudron incarne les enfers dans les représentations chrétiennes, qu'il s'agisse par exemple de la Divine Comédie de Dante (vers 1310) ou du Jugement dernier de Fra Angelico (vers 1430). A partir de la Renaissance, le thème de la marmite se détache de la problématique du Salut (la purification) pour retrouver celle de l'eucharistie (la souillure papale) : il apparaît alors dans des textes profanes (les contes du géant Gargantua), et il est parfois associé à la critique traditionnelle du moine, comme dans les Songeries drolatiques de Pantagruel $(1565)^{5}$. Dans le cadre des conflits confessionnels, ces deux héritages convergent pour faire du moine un glouton insatiable, un être hypocrite qui scandalise la divinité en détournant les préceptes chrétiens à son profit terrestre (pauvreté, piété, charité).

7 Cet anticléricalisme, bien représenté par les textes de Rabelais ${ }^{6}$, n'est pas incompatible avec la croyance chrétienne, et c'est bien là toute la difficulté : la critique des clercs peut venir tant de l'évangélisme (Erasme, Lefebvre d'Etaples) que du calvinisme (Théodore de Bèze, Simon Goulart). C'est ce qui explique que chaque camp ait pu s'en emparer, en retournant la critique ou en intégrant les attaques de l'adversaire. Nous avons alors pu constituer un corpus à la fois catholique et protestant, centré sur les années 1560.

\subsection{La constitution du corpus}

$8 \quad$ Le premier texte retenu est les Satyres chrestiennes de la cuisine papale, texte calviniste anonyme publié à Genève en 1560 et attribué à Théodore de Bèze par Charles-Antoine Chamay. Dès l'année suivante paraît une Comédie du pape malade tirant à la fin (1561), un texte beaucoup plus court et moins inventif, mais qui présente à nos yeux l'intérêt de reprendre immédiatement le motif de la marmite. S'y ajoutent deux opuscules protestants lyonnais, La desolation des freres de la robe grise, pour la perte de la marmite, qu'est renversée (1562), ainsi que La Polymachie des marmitons ou la gendarmerie du pape, en laquelle est amplement descrite l'ordre que le pape veut tenir en l'armée qu'il veut mettre sus pour l'enlèvement de sa marmite (1563). Enfin, certains ouvrages de Thomas Beaux-Amis, célèbre polémiste catholique, prennent place dans le corpus : La marmite renversee et fondue, de laquelle nostre Dieu parle par les saints prophetes (Paris, 1562 ?), texte repris en partie en 1568 et 1572 sous le titre Resolution sur certains pourtraits \& libelles, intitulez du nom de Marmitte, faussement imposé contre le Clergé de l'Eglise de Dieu (Paris, 1568). Nous ajoutons certaines gravures ayant pour thème principal la marmite, comme celles qui figurent dans le Recueil des pièces qui regardent la Ligue de Pierre de L'Estoile, c'est-à-dire les deux pièces ligueuses La cruauté en faisant mourir les catholiques ainsi que La marmitte renversee des huguenots, Politiques, Atheistes, Espernonistes, Libertins (1589), auxquelles il convient d'ajouter des estampes comme Le renversement de la grand marmitte (1585).

Trois remarques s'imposent. La première concerne le statut de ces textes, le plus souvent des pamphlets satiriques et anonymes diffusés à partir de presses qu'il est difficile d'identifier ${ }^{7}$, mais qui s'inscrivent dans une actualité brûlante et ont une durée de vie assez courte. D'autre part, le thème de la marmite ne disparaît pas après 1600 , et 
Frank Lestringant a indiqué que la marmite reste «l'une des images favorites de la satire calviniste jusqu'au début du XVIIe siècle ", comme l'indiquent les références à la « digne marmite » du pape, à la « rôtisserie papale » et au « potage renversé », dans le Tableau des differens de la religion de Marnix de Sainte-Aldegonde (Leyde, 1600-1605; Lestringant 1999 : 43). Enfin, il faut convenir du fait que ce corpus est exploratoire et qu'il ne recouvre certainement pas toutes les occurrences liées à ce motif. C'est dans ce cadre précis que l'on peut étudier l'émergence du terme de marmite comme insulte.

\section{La construction discursive de l'insulte}

Nous proposons de retracer le parcours de l'insulte dans des textes et gravures relevant de discours polémiques précisément situés. Nous rendrons compte de l'émergence, du développement et de la diffusion d'une insulte au sein d'une situation spécifique, afin de mettre en évidence des phénomènes d'interdépendance. Nous retracerons le parcours de cette insulte avant d'analyser les réalisations discursives privilégiées.

\subsection{Trois étapes dans la construction discursive de l'insulte}

11 L'utilisation du nom «marmite » dans le cadre des polémiques religieuses est perçue très tôt comme insultante pour la communauté catholique. L'étude du corpus permet de mettre en évidence la construction discursive de cette insulte. Il semble difficile, dans le cadre de ce travail, d'en affirmer une origine certaine ainsi qu'une évolution claire : il est en effet possible (mais difficilement vérifiable) que le mot "marmite " circule, en particulier dans les pratiques orales, comme insulte dès avant 1560 . Néanmoins, les textes ont selon nous des statuts différents, permettant de cerner des étapes dans le processus de construction de l'insulte: Les Satyres chrestiennes apparaissent dans cet ensemble comme l'un des lieux discursifs possibles de son élaboration. Le texte, en associant étroitement le motif de la marmite à une critique historiquement située de l'institution papale, tournée en particulier vers le thème de l'eucharistie, va constituer l'une des matrices possibles de la création de l'insulte en orientant les formes et les significations possibles de ce terme. L'ensemble du corpus permet d'observer ensuite ce que nous appellerons un déploiement de cette insulte, par une textualisation qui génère un certain nombre de variations dans les formulations. Enfin, la réappropriation du terme par les catholiques dans les deux textes de BeauxAmis et dans la gravure de 1585 constitue une troisième étape qui réoriente et renouvelle formes et significations.

\subsubsection{Les Satyres chrestiennes comme matrice possible de la marmite comme insulte}

Dans les Satyres chrestiennes, la marmite n'apparaît que comme l'un des motifs d'une allégorie donnant à voir une représentation plus globale mais partiale de l'univers papal. Le terme lui-même n'apparaît qu'une seule fois dans le pamphlet, au début de la satyre III (v. 14-25) :

Cordelieres, caymandieres,

Converses, vrayes vivandières,

Sçavent de la deesse Bonne,

Les secrets mieux que la Sorbonne.

Argumentation et Analyse du Discours, 8 | 2012 
De la les vivandiers convers

Ameinent chariots couverts

Pour remplir les larges marmites

Là pres sont marmitons hermites

Qui les pots brusquement escument.

Carmes s'escarmouchans, presument

Qu'ils sont bien le faict de souillars

Augustins, rustres et gouillars [...].

13 papauté : dans la structure de l'œuvre, la découverte de la vérité cachée, le «pot[s] aux roses » (satyre I, v. 80), est matérialisée par le cheminement du narrateur qui progresse de l'extérieur, le jardin, vers l'intérieur, la cuisine, dont le centre lui-même est constitué par les marmites. On peut ainsi percevoir une représentation des lieux comme constituée de cercles concentriques aboutissant aux centres vertigineux des marmites. Les verbes utilisés dans la satyre III, caractérisés par le trait "dynamique " («apportent », v. 10 ; «ameinent », v. 19), rendent compte de l'attraction généralisée des humains, narrateur et lecteur compris, vers les marmites. Si elles ne font donc elles-mêmes l'objet d'aucune caractérisation dégradante ou insultante, c'est essentiellement par métonymie qu'elles acquièrent dans le texte ces valeurs dépréciatives : tous ceux qui s'activent autour d'elles sont décrits en termes péjoratifs, comme le montrent par exemple les termes de «souillars» (malpropre) ou de «gouillars» (glouton) dans l'extrait relevé plus haut. Relevons en outre le rôle plus particulier du terme "marmitons ", déjà attesté dans le sens insultant d'« hypocrite » dans Pantagruel (1532) de Rabelais8. C'est ici le dérivé qui réactive «marmite » comme insulte, en rétablissant le lien avec le sens étymologique du terme. Selon le Dictionnaire historique de la langue française, le nom "marmite» est en effet construit par substantivation de l'adjectif "marmite», attesté au 13e siècle dans le sens $d^{\prime}$ ' hypocrite $»$. De manière plus précise, on peut supposer que le rapprochement cotextuel entre « marmite » et « marmitton » réactive le sens évaluatif du substantif, bien attesté en Ancien français mais vraisemblablement estompé en Moyen français ${ }^{10}$.

Dans cette satyre, si l'on admet que la cuisine papale désigne l'ensemble de la curie romaine au 16e siècle, avec en son centre le « grand ventru » papal attisant le feu, c'est toute l'activité ecclésiastique romaine qui est moquée. Par ces désignations de "gouillars » et "marmittons » se trouve réactivée la critique traditionnelle des vices associés au corps ecclésiastique que le terme «marmite» vient synthétiser : l'hypocrisie, l'ivrognerie et la gourmandise («Jacobins, bons buveurs», "gras coquins » : satyre III, v. 33 et 37), la saleté (« souillars » : v. 24) ou encore la luxure (par le rapprochement paronymique entre «cordeliers» et «bordeliers" par exemple: v. 31 et 32). Mais plus encore, cette critique rejoint les propos de Calvin contre les dissimulateurs, qui se «couvrent du manteau de vraie religion», qui «font les dévotieux ", bref qui feignent de vivre religieusement. Pour le réformateur, cette attitude de «nicodémite» est une gigantesque hypocrisie organisée depuis Rome ${ }^{11}$. Là encore, la marmite est au carrefour des dénonciations habituelles.

Le texte cependant va plus loin que cette critique traditionnelle de l'Eglise. Ce qui est nouveau, c'est la dénonciation, à travers les moines, de toute la hiérarchie (évêques, cardinaux), des usurpations de fonctions (prêtres ignares), du système des indulgences (rachat des péchés), des superstitions (processions, reliques, pèlerinages), mais surtout de manière privilégiée de l'eucharistie (la transsubstantiation). Or, tous ces aspects sont 
précisément ceux dont Luther puis Calvin s'emparent à partir de 1517 pour le premier (95 thèses contre les indulgences à Wittenberg) et de 1536 pour le second (édition bâloise de l'Institution de la religion chrétienne). Au-delà de la dimension satirique, il y a donc bien une véritable lecture théologique de la marmite, qui met en jeu les fondements des différences confessionnelles et révèle les points de tensions.

\subsubsection{Les autres occurrences du mot « marmite » dans le corpus : le déploiement de l'insulte}

Dans les autres textes du corpus, la marmite apparaît comme le motif principal et l'usage du terme est toujours directement insultant. L'utilisation de l'article défini à valeur de notoriété, soit dans la première occurrence du terme au sein d'un des textes, soit dans le titre des gravures ou des libelles, rend compte de la circulation de ce syntagme. Il en est ainsi du texte protestant intitulé Désolation des frères de la robe grise, pour la perte de la marmite, qu'est renversé, qui paraît à Lyon deux ans après la publication des Satyres chrestiennes, en $1562^{12}$. Le terme de "marmite", associé au monde ecclésiastique, n'est absolument pas explicité, ce qui laisse supposer qu'il est suffisamment clair pour la communauté chrétienne de Lyon. La dimension insultante peut se manifester par un commentaire métadiscursif, comme dans la Résolution de Beaux-Amis (1568). Ainsi l'Avis au lecteur contient-il une première catégorisation au sujet de l'emploi par les huguenots du syntagme « marmite papale »:

L'Eglise nouvelle n'a eu crubescence par animadversions excessives, sans quelque couleur de la saincte ecripture, appliquer un nom trop enorme à l'eglise romaine et au clergé d'icelle, l'appelant Marmitte papale, et en images n'ont craint exhiber cette malicieuse reproche.

17 Le groupe nominal, entrant pour lui dans la catégorie du "reproche», de "l'animadversion excessive » ou encore de "l'application d'un nom trop enorme", relève plus précisément de la calomnie. Ce terme même est d'ailleurs employé explicitement à la fin du texte, tout comme celui de «blasphèmes ", d'«injures » et d'« opprobres »:

Les [dignitez ecclesiastiques] ayant brisé et rompues par calomnies et blasphemes execrables, ils les ont mises ainsi qu'en une marmite, comme tesmoignent les libelles d'injures, qu'ils ont fait imprimer contre le Pape, Cardinaux, Evesques, Prestes, Moines et mendians. Et non contens de ce, ils ont fait depeindre et imprimer diverses pourtraitures de marmites, qu'ils appellent papales. Mais tout bon esprit verra que de leur part ne sont qu'opprobres.

Si les injures désignent ici de manière générale toutes les attaques verbales contenues dans les pamphlets des réformés, elles réfèrent également à ces libelles dont il a été question dans la Résolution, c'est-à-dire aux ouvrages contenant le nom même de marmite.

La dimension insultante du terme peut également apparaître textuellement. Il peut s'agir d'une association co-textuelle, mettant par exemple en relation la marmite avec un vice, comme dans la gravure Le reversement de la grande marmite (1561), dont le cartouche associe marmite et hypocrisie : «la Verité a du tout renversée / L'hypocrisie et la marmitte aussy». La valeur insultante du terme "marmite» dans toutes ces occurrences passe par une textualisation qui favorise un certain nombre de formulations. C'est ce que nous avons appelé le déploiement discursif de l'insulte. 


\subsubsection{Le retournement de l'insulte : le texte de Thomas Beaux-Amis}

Ce déploiement se poursuit dans les textes du catholique Thomas Beaux-Amis, et ses ouvrages proposent également un certain nombre de formulations insultantes. Néanmoins, on propose de considérer les différentes versions de La Résolution comme une étape nouvelle dans la construction discursive de l'insulte. En effet, même si dans les autres textes les significations du terme peuvent être diversifiées, elles renvoient toutes à une critique de l'univers papal et catholique. A l'inverse, lorsque le terme est repris dans un contexte polémique, au sein d'un écrit catholique, il change de signification, tout en gardant une valeur d'insulte : il est renvoyé à l'église réformée, au terme d'une réinterprétation des Ecritures. Une lecture inversée de la Bible permet ainsi de constituer un argument d'autorité pour administrer une preuve de la volonté divine. Le titre du pamphlet de Beaux-Amis rend compte de ce retournement: Résolution sur certains pourtraits et libelles, intitulez du nom de marmitte, faussement imposé contre le clergé de l'Eglise de Dieu. Par laquelle est prouvé par le discours de l'escriture saincte, et l'expresse parole de Dieu, le nom de Marmitte enflambee, estre propre à la nouvelle Eglise.

La dimension dialogique et polémique est ici explicitée, puisque le texte se présente comme répondant aux différents "libelles» et "pourtraits» portant le nom de marmite. Mais ce qui donne à l'insulte une nouvelle portée réside dans le retournement du motif, la réattribution du nom de marmite à l'Eglise réformée. Il s'agit là d'un processus discursif généralisé pendant les guerres de religion, consistant à employer les mots de l'autre pour les lui retourner. Ainsi en va-t-il des "séditieux», des « anabaptistes », des « jézabélistes » etc., qui désignent alternativement les catholiques ou les calvinistes ${ }^{13}$. Mais cette utilisation de la langue de l'ennemi pour la lui renvoyer est nouvelle dans le parcours spécifique de l'insulte. Elle permet à la fois une continuité dans l'emploi du terme mais instaure aussi une rupture, dans la mesure où elle favorise une réorientation des référents et éventuellement des formes textuelles. Ce sont ces différentes formulations intégrant le terme de «marmite " que nous allons à présent étudier.

\subsection{Les variations dans la mise en texte du nom « marmite »}

L'étude du parcours des différentes formulations met en évidence des variations qui se stabilisent autour de certains patrons redondants. Deux types de constructions retiendront particulièrement l'attention: le terme "marmite " avec une expansion adjectivale ou un complément du nom, et le terme "marmite» dans une structure passive.

\subsection{1. « marmite » + expansion adjectivale ou complément de nom}

Si le terme de « marmite » apparait parfois seulement avec un déterminant défini, on le rencontre très fréquemment avec une expansion nominale, de type adjectival ou complément de nom. On étudiera successivement les deux types de construction en s'appuyant sur quelques occurrences.

24 1. «marmite + adjectif »: il peut s'agir soit d'adjectifs qualificatifs, soit d'adjectifs relationnels.

Les adjectifs qualificatifs: on relève des occurrences comme «larges marmites » (Les Satyres chrétiennes) ou « les marmites grasses " (La Comédie du pape malade et tirant à sa 
fin). Ces caractérisations doivent toujours être interprétées par transposition métaphorique. Même si, dans la première occurrence, l'adjectif «large" semble préciser simplement l'aspect extérieur des marmites et ne transgresse pas les règles de sélection catégorielle entre un nom et un adjectif, il fonctionne avant tout de manière métaphorique pour caractériser un univers papal puissant et engloutissant. Cette dimension est encore plus perceptible dans le deuxième exemple, puisqu'on observe bien une transgression des règles de sélection. Ainsi, l'adjectif « grasses » relève soit de la métonymie (le contenu pour le contenant) soit de l'hypallage, attribuant à la marmite une caractérisation dépréciative traditionnellement réservée au clergé et relative à la gloutonnerie. De manière générale, les deux catégories sémantiques des adjectifs qualificatifs les plus fréquentes sont celles de la grande dimension et du sens gustatif.

Les adjectifs relationnels : on relève des occurrences comme "marmite papale », mais aussi « marmite claustrale » ou encore " marmite presbitériale ». Dans ces occurrences, l'ambiguïté relationnelle constitutive de ce type d'adjectif n'a pas pour fonction de cacher ou d'éviter l'explicitation d'un type de relation entre le déterminant et le déterminé. Il semble que la fonction essentielle de ces constructions est avant tout la mise en relation elle-même, le rapprochement entre ce que le nom de marmite peut signifier de dégradant et l'univers qui lui est associé par cette construction syntaxique. Celle-ci manifeste davantage une indifférence au type de relation établie (elles sont toutes acceptables) pour se focaliser sur la relation elle-même, puisque c'est cela même qui est source de dénomination dégradante ;

2. «marmite + groupe prépositionnel » : il s'agit dans tous les cas de compléments de nom construits avec la préposition «de »: " marmite du pape », ou encore «marmite renversée des huguenots, politiques, Atheistes, esperonistes, libertins», etc. Dans ce cas encore, ce qui est privilégié n'est pas le type de relation mais la mise en relation elle-même. L'énumération qui prévaut dans cette dernière occurrence mérite un commentaire supplémentaire. En effet, ce procédé stylistique permet ici d'exprimer à la fois la multiplicité, opposée à l'unicité de la vérité et l'absence de hiérarchie. Aux yeux des ligueurs, d'innombrables menteurs se retrouvent jetés indistinctement dans la marmite.

\subsection{2. " marmite » + structure passive}

Le deuxième type de construction concerne l'association de "marmite " dans une structure passive. Il peut s'agir soit de phrases passives sans complément, soit de l'association de « marmite » avec un participe passé.

- Les phrases passives sans complément d'agent: on trouve des occurrences comme « Perte de la marmite, qui est renversé » ou la « marmite sera répandue »;

- « marmite » + participe passé : on trouve des occurrences comme « Marmite renversée et fondue » ou encore « marmite enflambée ».

Même si le corpus comporte des occurrences avec des formes actives de type V+ marmite ( «la Verité a du tout renversée / L'hypocrisie et la marmitte aussy »), ce sont les constructions passives qui dominent. Cette redondance doit être relevée et analysée. D'une part, il s'agit de structures employant des verbes perfectifs (renverser, fondre), qui peuvent, que l'auxiliaire être soit exprimé ou non, correspondre à une lecture processive (marmite renversée $=$ marmite qui est renversée, la marmite qu'on renverse) ou une lecture résultative (la marmite qui a été renversée) relevant alors de l'aspect 
accompli. D'autre part, la valeur sémantique des verbes exprime un procès de destruction (fondre, enflamber) et de renversement. Même si l'on observe des variantes quant au sujet grammatical («marmite » mais aussi « chaudron»), même si l'on note, comme ci-dessus, d'autres patrons syntaxiques associant «marmite » et des mots de la famille de "renverser", on remarque également une prédilection pour «marmite + renversée » au participe passé qui demande un commentaire particulier.

Il y a une signification théologique à cette prédominance. Elle revient à considérer l'histoire de l'Eglise chrétienne comme une longue attente de libération de la servitude papale (ce que Luther appelle "la captivité babylonienne de l'Eglise», 1520). Le renversement de la marmite, dans la lecture résultative, c'est à la fois la réalisation de cette promesse faite par Dieu à son peuple et l'expression du soulagement des fidèles. Mais on doit reconnaitre la force théologique de la lecture processive: dans cette théologie de la libération, le phénomène est strictement contemporain, au sens où les réformateurs annoncent un processus à la fois imminent et en cours. Il ne s'agit donc ni d'une parole historique dressant l'état d'une action passée (théologie du soulagement), ni d'une parole prophétique imaginant un avenir meilleur (théologie de l'attente), mais bien d'une parole narrative décrivant une chute en acte (théologie de la révélation). Cette interprétation processive est confortée par la gravure La Marmitte renversee des huguenots, Politiques, Atheistes, Espernonistes, Libertins, sur laquelle le chaudron est en déséquilibre, poussé et tiré d'un côté, retenu de l'autre.

\section{Insulte et argumentation}

Le terme de "marmite » qui émerge comme insulte dans la sphère religieuse au $16 \mathrm{e}$ siècle est utilisé dans une fonction argumentative. Cette dimension est permanente dans le corpus, mais peut reposer sur des processus distincts. Nous étudierons trois de ces fonctionnements.

\subsection{L'utilisation du terme " marmite » au service d'une entreprise de démystification}

30 Théodore de Bèze, dans les Satyres chrestiennes de la cuisine papale, n'utilise pas d'arguments théologiques face à ses adversaires. Comme le remarque Charles-Antoine Chamay dans l'introduction à l'édition critique de cette œuvre (2005:XI), cette forme d'argumentation est surprenante dans le cadre de la polémique calviniste de l'époque :

[...] tandis que les disputations chrétiennes de Pierre Viret et la majorité des œuvres de polémique calviniste du temps accordent une place majeure à l'apologétique, à la justification théologique, les Satyres chrétiennes de la cuisine papale ne semblent guère s'en occuper.

C'est que se développe dans ce texte une forme de la rhétorique calvinienne utilisant la force didactique de la satire. Ce recours à la satire n'a pas pour fonction principale une critique personnelle ${ }^{14}$, mais vise l'ensemble de la papauté dans une entreprise de démystification: dans le prolongement de ce que nous venons de voir au sujet de la "théologie de la révélation », il s'agit de déchiffrer, révéler, dévoiler l'évidence de la tromperie en l'exhibant aux yeux de tous. Même si l'« instruction » prend ici d'autres voies, même si l'on s'écarte de la recommandation de Calvin de " tenir le moyen » dans l'utilisation de la langue ${ }^{15}$, la dimension didactique de la satire est primordiale, puisqu'il 
s'agit de faire tomber les masques qui voilent le rapport au vrai, afin d'éviter de nouvelles conversions au catholicisme une fois déclenchées les guerres de religion: «de sorte que non seulement [la vérité] peut être reçeuë par demonstrations et graves authoritez, mais aussi sous la couverture de quelque facetie» (Bèze 2005: 6). La production de fausses confessions, dans ce cadre, participe du même objectif. Certains ministres réformés font en effet paraitre ce genre d'écrits révélant la véritable nature des sacrements catholiques. Hugues Sureau, réfugié à Genève puis à Heidelberg, a même produit plusieurs textes de ce type au fur et à mesure de ses conversions successives : en 1573 paraît ainsi une Confession et recognoissance d'Hugues Sureau, dit Du Rosier, touchant sa cheute en la papauté et les horribles scandales par luy commis ${ }^{16}$.

Dans le cadre de ce jeu de masques, le choix des mots et des figures utilisés dans les textes est d'une importance cruciale. Dans les Satyres chrestiennes, la multiplication des paronomases, des jeux sur le signifiant servent la même cause : les mots usités, les mots officiels, cachent d'autres mots, d'autres signifiants, révélateurs d'une réalité plus sordide, diabolique ou simplement fausse. Le langage catholique est lui-même un faux langage qu'il s'agit de déchiffrer. Il peut s'agir par exemple de donner d'abord le mot usuel pour ajouter ensuite un autre terme, plus conforme: «Le pape ou happe, si tu veux ». Par le recours à une modalisation autonymique interlocutive, le poète entend ainsi mettre en évidence la non-coïncidence entre le mot et la chose, puis son remplacement par un autre terme considéré comme plus adapté mais franchement insultant $t^{17}$.

33 L'utilisation du terme de marmite dans les textes du corpus participe du même projet de démystification. Il fait en effet surgir dans le monde spirituel une réalité vulgaire, perçue comme dégradante. Dans le texte de Théodore de Bèze, la marmite est associée aux divers membres du monde ecclésiastique : béguines, cordelières, jacobins, ermites, augustins, etc. La proximité voire la confusion entre le fonctionnement des ordres religieux et les lieux dégradants de la marmite se matérialise alors par le rapprochement co-textuel de termes relevant habituellement de champs lexicaux éloignés. L'utilisation répétée de la structure "marmite + adjectif relationnel», soulignée plus haut, relève du même objectif. Ce procédé sera repris par les autres textes $\mathrm{du}$ corpus avec des associations permettant par exemple une même désacralisation du camp adverse. Ainsi en va-t-il de la gravure La Marmitte renversée des huguenots, politiques, Atheistes, Esperonistes, Libertins (1589). Issue du courant des catholiques radicaux (les ligueurs) qui sont opposés à la fois aux protestants et aux catholiques fidèles aux rois, elle rapproche les calvinistes, les royalistes, les membres de la cour (comme le duc d'Epernon, particulièrement détesté) et les incroyants de tous ordres (athéistes, libertins). Quelles que soient les différences profondes qui distinguent ces groupes de personnages hétéroclites, le titre de la gravure permet de les confondre en une longue énumération et de les avilir par la mise en relation avec le terme « marmite ».

On voit donc que la démystification à l'œuvre dans les différents processus de rapprochement repose sur la potentialité dépréciative du mot « marmite ». Or, il s'agit d'un terme qui, fonctionnant comme métaphore ou comme métonymie, intègre et cristallise des significations très variées et très riches. 


\subsection{Les ressources argumentatives de la marmite comme métaphore et métonymie} l'époque étudiée tient en grande partie à son utilisation argumentative comme métonymie ou comme métaphore. Comme métonymie, tout d'abord, les différentes reprises du terme peuvent ainsi intégrer les signifiés variés liés aux univers susceptibles de lui être associés. Dans le Jugement dernier de Fra Angelico par exemple, la marmite est le lieu où cuisent les réprouvés avant d'être dévorés par le démon. Dans les Songeries drolatiques de Pantagruel (1565), la marmite est ce qui donne corps à des personnages à la fois ridicules et monstrueux, proches des géants des contes populaires, porteurs d'attributs culinaires (fourchettes, cuillères, casseroles, etc.). L'interprétation joue alors sur l'appel à des connaissances encyclopédiques et à une mémoire discursive dont les contours sont flous mais surtout sont susceptibles de se superposer. Si l'on peut supposer une communauté discursive stable (quoique faible) capable de référer le terme de marmite aux textes bibliques, d'autres éléments discursifs peuvent lui être associés et superposés (notamment chez Rabelais, comme nous l'avons vu plus haut). Il en résulte une richesse des interprétations possibles, fondée non pas sur l'explicitation mais sur l'appel implicite à une critique déjà admise (la critique des moines dans Gargantua, 1534). La richesse de l'utilisation du terme de «marmite ", présente dans le contexte de l'enfer et dans celui de la cuisine au titre de la dévoration, est justement de pouvoir fonder la critique théologique de l'eucharistie sur la mémoire de textes où cette critique n'apparaît pas. Parce que le motif de la marmite est suffisamment large, parce qu'il appartient à des domaines d'expérience distincts et relève de mémoires discursives variées, il permet de rassembler ce qui est différent et de condenser des éléments de critiques de nature hétérogène. Il se trouve au carrefour de traditions scripturaires (Ezechiel, Jérémie), populaires (les contes de géants), littéraires (Rabelais) et picturales (Fra Angelico).

36 Le terme de "marmite " est également caractérisé comme insulte relevant de la métaphore. Celle-ci met en œuvre des rapports analogiques entre divers domaines d'expérience. Mais dans la mesure où la relation sémique non seulement n'est pas explicitée mais dépend d'une contextualisation toujours nouvelle, la métaphore permet le développement renouvelé de significations. En ce qui concerne la marmite, l'exploitation de traits très généraux comme la forme ou la relation contenant/ contenu, par exemple, permet une grande plasticité dans les rapprochements :

- pour ce qui est de la forme extérieure de la marmite, c'est la rotondité qui peut être sélectionnée pour mettre en œuvre des rapprochements (comme le ventre, par exemple), mais d'autres formes sont parfois préférées, comme l'ovale, autorisant le rapprochement entre marmite et cloche ou tiare renversées. Dans ce cas, le retournement effectif matérialise l'inversion, détournant les emblèmes de l'institution ecclésiastique pour en faire les instruments du supplice des chrétiens. Dans cette marmite en forme de tiare ou de cloche, c'est la dimension anthropophagique de la papauté qui est dénoncée. On retrouve donc une nouvelle fois la cristallisation des tensions autour de la question de l'eucharistie : les catholiques, en mangeant la chair du Christ et en buvant son sang, ne se distinguent pas vraiment des cannibales que l'on vient de découvrir outre-atlantique. C'est du moins ce qu'affirment les pamphlets luthériens (Flugschriften) du milieu du siècle ; 
ce qui est de la distinction contenant/contenu, la marmite est susceptible de renvoyer à l'association de contenants et contenus très variés, concrets et/ou abstraits : le ventre, la ville maudite, l'Eglise, les chrétiens, la doctrine catholique et même les ouvrages pamphlétaires. Cette plasticité est particulièrement exploitée par Thomas Beaux-Amis, qui reprend la signification de la marmite donnée dans Ezechiel mais en y ajoutant un sens supplémentaire original. La marmite est à la fois la cité des chrétiens qui se consument et l'ensemble des ouvrages contenant les injures faites contre l'Eglise romaine :

Ayant brisees [les dignitez ecclesiastiques] et rompues par calomnies et blasphemes execrables, ils les ont mises ainsi qu'une marmitte, comme tesmoignent les libelles d'injures, qu'ils ont faict imprimer contre le Pape, Cardinaux, Evesques, Prestres, Moines, et Mendians. Et non contents de ce ont faict depeindre et imprimer diverses pourtraitures de Marmitte, qu'ils appelent papale (Beaux-Amis $1568: 15$ ).

La richesse du terme de "marmite ", une nouvelle fois, est telle qu'elle sert des projets complexes. Cette remarque est confirmée par la question du renversement.

\subsection{Retournement de l'insulte et argumentation}

La spécificité du parcours de l'insulte construite autour du terme de "marmite" provient enfin de la dimension dialogique que l'on perçoit en particulier dans le texte de Beaux-Amis, renvoyant explicitement l'insulte à l'adversaire. Dominique Lagorgette a étudié certaines formes de retournement de l'insulte à son auteur ${ }^{18}$, en particulier dans les cas où le destinataire s'approprie l'insulte, accepte et assume l'identité dégradante, afin d'atteindre son auteur par un mécanisme de renvoi. Le processus de retournement est ici différent, dans la mesure où il s'agit d'attribuer à l'adversaire politique le nom insultant. Ce renvoi apparait dans le titre de La Résolution, mais également dans d'autres parties du texte, en particulier dans l'avis au lecteur :

[a] ce qu'il soit manifeste combien est inique ceste canillation, j'ay assemblé en ce petit traité toutes les Marmites, desquelles est parlé en la Bible soubs figure: montrant cuidemment qu'elles sont entendues des sectes heretiques, et que le nom de Marmite, selon l'intelligence de l'escriture, doit estre imputé à leur eglise nouvelle.

41 On le voit, le renvoi de l'insulte à l'insulteur s'opère par une tentative de changement de signification qui passe par un processus de redéfinition du terme " marmite ", dont Perelman et Olbrechts-Tyteca (2000: 282 et suiv.) ont souligné l'importance et la fréquence dans l'argumentation. Il est difficile, dans le cadre de cet article, de décrire en détail ce travail de redéfinition, mais on peut en indiquer succinctement les étapes. Partant d'extraits du texte biblique mentionnant la marmite dans un épisode narratif (Ezechiel, Jérémie, mais aussi Michée, etc.), l'auteur procède à une exégèse allégorique permettant de donner une équivalence à chacun des éléments du récit : «Recherchons les significations de toutes ces choses, puis nous viendrons à notre conclusion ». Ainsi les Israelites qui s'opposent à Moïse et qui regrettent les marmites d'Egypte sont-ils assimilés aux huguenots qui s'en prennent au pape et regrettent la liberté de vivre dans le péché19. La marmite connaît dans ce passage un parcours qui permet d'en faire évoluer la signification: elle désigne d'abord l'idée de «liberté de manger de toutes viandes, [...] vivre comme des Epicuriens ", puis par métonymie, ceux qui partagent cette idée, jusqu'à désigner l'Eglise qui les rassemble : «Est-il pas donc manifeste, que

Argumentation et Analyse du Discours, 8 | 2012 
veu que la Marmitte peult en ce passage signifier l'Eglise libertine, que de ce nom proprement les nouveaux Evangelistes seront denommez?»

La force argumentative de la redéfinition tient ici à deux points : d'une part, celle-ci est étayée par le texte biblique, autorité indiscutable pour ceux à qui le texte est adressé et qui remplit la fonction de prémisses. D'autre part, le terme de marmite, même travaillé par une définition nouvelle, garde la valeur d'insulte, déjà construite discursivement par les occurrences précédentes.

\section{Conclusion}

Les usages injurieux du terme "marmite » connaissent un essor important dans les années 1560. Dans les polémiques opposant catholiques et protestants, les pamphlets se répondent les uns aux autres et utilisent toutes les ressources interprétatives de ce motif. La marmite est successivement centre de la cuisine papale, chaudron infernal du diable, figuration de l'eucharistie, révélateur de la mystification de la messe, espace de retournement des valeurs et des institutions. A ce titre, comme nous l'avons vu, la marmite est au carrefour de traditions scripturaires, littéraires, populaires et picturales.

Mais tous ces sens n'ont pas la même valeur et ne justifient pas la fréquence et la permanence de la «marmite » dans les polémiques. La marmite concentre l'attention sur le cœur de l'opposition entre catholiques et protestants : l'eucharistie. Elle permet donc de mettre en évidence l'actualité du texte biblique tout en dénonçant les dérives théologiques qui, selon les huguenots, ont progressivement conduit l'église à sa ruine.

\section{BIBLIOGRAPHIE}

\section{Corpus}

[Anonyme]. 1561. Comédie du pape malade tirant à la fin

[Anonyme]. 1562. La Desolation des freres de la robe grise, pour la perte de la marmite, qu'est renversee (Lyon)

[Anonyme]. 1563. La Polymachie des marmitons ou la gendarmerie du pape, en laquelle est amplement descrite l'ordre que le pape veut tenir en l'armée qu'il veut mettre sus pour l'enlèvement de sa marmite (Lyon : Jean Sangrin).

Beaux-Amis, Thomas. 1562. La marmite renversee et fondue, de laquelle nostre Dieu parle par les saints prophetes (Paris)

Beaux-Amis, Thomas. 1568 et 1572. Resolution sur certains pourtraits \& libelles, intitulez du nom de Marmitte, faussement imposé contre le Clergé de l'Eglise de Dieu (Paris : Jerôme de Marnef \& Guillaume Cavellat) 
[Bèze, Théodore de]. 1560. Satyres chrestiennes de la cuisine papale, éd. Charles-Antoine Chamay. 2005 (Genève : Droz)

Calvin, Jean. 1536. Institution de la religion chrétienne, éd. Jean-Daniel Benoist, 4 vol. 1957 (Paris : Vrin)

Calvin, Jean. Petit traité de la Sainte Cène, éd. Harald Chatelain \& Pierre Marcel. 2008 (Lyon : Olivetan)

Désiré, Artus. 1560. Contrepoison des cinquante-deux chansons de Clément Marot (Paris : Pierre Gaultier)

Gravures :

[Anonyme]. 1585. Le Renversement de la grand marmite (BNF, Estampes Qb 1)

[Anonyme]. 1589. La cruauté en faisant mourir les catholiques (Pierre de l'Estoile)

[Anonyme]. 1589. La Marmitte renversee des huguenots, Politiques, Atheistes, Espernonistes, Libertins (Pierre de l'Estoile)

\section{Etudes :}

Amossy, Ruth. 2000. L'Argumentation dans le discours. Discours politique, littérature d'idées, fiction (Paris : Nathan Université)

Angenot, Marc. 1980. La Parole pamphlétaire. Typologie des discours modernes (Paris : Payot)

Authier-Revuz Jacqueline. 2005. Ces Mots qui ne vont pas de soi. Boucles réflexives et non-coïncidences du dire (Paris : Larousse)

Bernard, Mathilde. 2010. Écrire la peur à l'époque des guerres de Religion. Une étude des historiens et mémorialistes contemporains des guerres civiles en France (1562-1598) (Paris: Hermann)

Carbonnier-Burkhard, Marianne. 2009. "Calvin dans des recueils de prières nicodémites?", Bulletin de la société de l'histoire du protestantisme français, vol. 155, 129-151

Crouzet, Denis. 1990. Les Guerriers de Dieu. La violence au temps des troubles de religion, vers 1525-vers 1610 (Seyssel : Champ Vallon)

Krieg-Planque, Alice. 2003. La notion de " formule » en analyse du discours. Cadre méthodique et méthodologique (Besançon : Presses universitaires de Franche-Comté)

Lagorgette, Dominique. 2008. " "Les moutons noirs contre-attaquent" : réappropriation du dire de l'autre et effet "boomerang" - les insultes aux "nonistes", communication à la journée d'étude "L'insulte : aspects linguistiques ; circulations et réappropriations », organisée par Thomas Bouchet et Caroline Mellet, Centre Georges Chevrier, Dijon

Lestringant, Frank. 1999. « Le Cannibale et la Marmite », Gomez-Gérault, Marie-Christine, Frank Lestringant, (éds), D’encre de Brésil. Jean de Léry, écrivain (Orléans : Paradigme), 39-63

Mellet, Paul-Alexis. 2012. « Monarchomaques, Anabaptistes, Jézabélites ! Les polémiques des guerres de religion », Crémoux, Françoise \& Fournel, Jean-Louis (éd.). La langue de l'Eglise et de l'Etat : guerres, régime, ordre (XVè-XVIIè s.) (Genève, Droz)

Mellet, Paul-Alexis. 2007. Les Traités Monarchomaques. Confusion des temps, résistance armée et monarchie parfaite (vers 1560-vers 1600) (Genève : Droz)

Parker, Charles H. 1993. «French Calvinists As the Children of Israel: An Old Testament SelfConsciousness in Jean Crespin's Histoire des Martyrs before the Wars of Religion ", The Sixteenth Century Journal, vol. 24, n² 2, 1993, p. 227-248 
Perelman, Chaïm et Olbrechts-Tyteca, Lucie. 2000. Traité de l'argumentation (Bruxelles : Editions de l'Université de Bruxelles)

Pitassi, Maria-Cristina et Daniela, Solfaroli Camillocci (éd.). 2010. Les modes de la conversion confessionnelle : autobiographie, altérité et construction des identités religieuses (XVIe-XVIIIe s.) (Firenze : Leo Olschki)

Postel, Claude. 2004. Traité des invectives au temps de la Réforme (Paris : Les Belles Lettres)

Rey, Alain (éd.). 2006. Dictionnaire historique de la langue française, 3 vol. (Paris : Le Robert)

Rosier, Laurence. 2006. Petit traité de l'insulte (Loverval : Editions Labor)

Szabari, Antónia. 2005. « Rabelais Parrhesiastes: The Rhetoric of Insult and Rabelais's Cynical

Mask », Modern Language Notes, 120, supplément, 84-123

\section{NOTES}

1. On trouvera dans son ouvrage La notion de " formule » en analyse du discours les principaux traits de définition de la formule.

2. Par exemple l'édit de Saint-Germain-en-Laye (août 1570), article $2:$ « Deffendant à tous noz subjectz [...] s'attacher, injurier ne provocquer l'un l'autre par reproche de ce qui s'est passé, en disputer, contester, quereler ny s'oultrager ou offenser de faict ou de parole ».

3. «Par ces inventions et autres sembables, Satan s'est efforcé d'espandre et mesler les ténèbres en la sacree Cène de Jesus Christ, pour la corrompre, depraver et obscurcir, a tout le moins afin que la pureté d'icelle ne fust retenue et gardee en l'Eglise » (Calvin 1536: 448). Voir surtout le Petit traité de la Sainte Cène de Calvin.

4. Paul-Alexis Mellet $2007: 238$.

5. Voir par exemple les Songeries drolatiques de Pantagruel (Genève : Droz), 2004, planches XXIII et XLIV.

6. Par exemple Gargantua (1534), éd. Mireille Huchon (Paris : Pléiade), chap. 39-45, p. 106-121.

7. Genève pour les Satyres chrestiennes.

8. Voir Antónia Szabari 2005.

9. Alain Rey $2006: 2143$.

10. Nous tenons à remercier chaleureusement nos collègues Annie Bertin et Sabine Lehman pour les informations précieuses qu'elles nous ont fournies au sujet de l'évolution sémantique du terme «marmite » entre l'Ancien français et le français de la Renaissance.

11. Voir à ce sujet Marianne Carbonnier-Burkhard (2009: 129-151). Le sens de "marmite» comme « hypocrite » semble donc s'être maintenu, comme le prouve également Le renversement de la grande marmite.

12. Une étude critique précise doit mentionner qu'il ne nous a pas été possible de retrouver un exemplaire original d'une version de cet opuscule. Nous avons utilisé la version qu'en donnent Cimber et Danjou dans leurs Archives curieuses de l'histoire de France. Sans remettre en cause le sérieux de leur travail de transcription, on doit donc prendre le titre qu'ils proposent avec prudence.

13. Voir à ce sujet P.-A. Mellet 2012.

14. Même si, d'après Charles-Antoine Chamay, le texte répond peut-être à Artus Désiré et Pierre Lizet.

15. Cité par Chamay 2005 : XIII

16. Voir par exemple M.-C. Pitassi et D. Solfaroli Camillocci 2010 et M. Bernard $2010: 175$ et suiv. 
17. Ajoutons bien sûr, dans cet exemple, le recours à la paronomase, qui permet, par le rapprochement des signifiants, de suggérer un rapprochement des signifiés.

18. Voir à ce sujet Lagorgette 2008.

19. Voir Charles H. Parker 1993.

\section{RÉSUMÉS}

L'article propose de montrer comment le motif de la marmite, courant dans la culture de la Renaissance, se spécialise dans un sens politique pour constituer une insulte dans le contexte précis de la polémique opposant pamphlets catholiques et protestants pendant les guerres de religion. En effet, le motif de la marmite, objet central de la cuisine, condense héritage biblique (Ezechiel XI et XXIV), tradition littéraire (Plaute) et culture populaire (telle que Rabelais s'en fait l'écho), pour constituer une critique des présumés péchés du clergé (concupiscence, gourmandise, pacte avec le diable, etc.). A travers l'étude de plusieurs pamphlets (Thomas BeauxAmis, Théodore de Bèze, etc.) se répondant dans le cadre d'une polémique centrée sur les rites chrétiens, nous mettons en évidence la dynamique discursive permettant la réappropriation du motif dans une valeur d'insulte. Nous étudions en particulier les processus argumentatifs utilisés au fil des pamphlets par chaque camp pour renvoyer l'insulte à l'autre. Il est alors possible de suivre le cheminement discursif de la construction d'une insulte. Il s'agit enfin de souligner la force argumentative de l'image satirique dont la fonction est bien sûr pour les deux Eglises de conforter la confession et pour les catholiques d'éviter la conversion au protestantisme.

This article shows how the motif of the cooking pot, recurrent in Renaissance culture, was, in the particular context of the polemics which raged between Catholic and Protestant pamphleteers during the French religious wars, converted into an elaborate political insult. A central element of culinary life, the cooking pot was also loaded with biblical heritage (Ezekiel 11 and 24), literary tradition (Plautus) and popular culture (such as that propounded by Rabelais), which made of it a concise symbol of the supposed sins of the clergy (concupiscence, gourmandise, pact with the devil, etc.). Through the study of several pamphlets (Thomas Beaux-Amis, Theodore Beza, etc.), which answer each other in the context of a polemic centred on Christian rites, we expose the discursive dynamics through which the motif of the cooking pot was appropriated as a political insult. We focus in particular on the argumentative processes employed by each camp in throwing the insult back onto their opponent - the study of this batting back and forth enabling us to trace the discursive construction of the insult. We also underline the controversial force of this satirical image, which was naturally associated by both churches with the consolidation of the practice of confession and employed by the Catholics as a tool against conversion to protestantism.

\section{INDEX}

Keywords : eucharist, French religious wars, insult, overturned pot, pamphlets

Mots-clés : eucharistie, guerres de religion, insultes, marmite renversée, pamphlets 


\section{AUTEURS}

\section{CAROLINE MELLET}

Université Paris Ouest Nanterre La Défense, Modyco UMR 77114

PAUL-ALEXIS MELLET

Université Cergy-Pontoise, CESR UMR 6576 